\section{Von Hitzewellen und Kühlflächen}

\author{
Seit Jahrzehnten wird über Nachhaltigkeit und die sich \\ daraus ergebenden Konsequenzen für Landnutzungen und \\ das jeweilige Planungssystem debattiert. Der globale \\ Klimawandel offenbart jedoch, dass das Planungssystem \\ Deutschlands noch weit davon entfernt ist, ökologisch \\ nachhaltig zu sein. Von Dietwald Gruehn
}

$\mathbf{Z}$ ur Bestimmung dessen, was nachhaltig sein soll, werden neben der ökologischen Dimension die soziale und die ökonomische Dimension als gleichrangig angesehen. Dabei darf allerdings nicht übersehen werden, dass die Tragfähigkeit ökologischer Systeme begrenzt ist und daher auch nur in beschränktem Maße verhandelbar ist. Dies bedeutet, dass ökologische Belange im Rahmen von planerischen Entscheidungen nicht in beliebigem Maße einer Abwägung zugänglich sind. Dennoch trifft genau dies auf die meisten Planungsinstrumente in Deutschland zu. In der Konsequenz ist in Deutschland ein Landnutzungsmuster entstanden, das in nicht unerheblichem Maße anfällig ist für zahlreiche Folgewirkungen des Klimawandels. Am Ende wird der Mensch selbst Betroffener dieser Entwicklung sein.

Bemerkenswert ist vor diesem Hintergrund, dass der Landschaftsökologe Langer die systematische Struktur dieses Prozesses bereits in den 1970er Jahren erkannt und in seiner Theorie des Systems „Mensch - räumliche Umwelt“ umfassend beschrieben hat (Langer 1978). Demnach dient die räumliche Umwelt dem Menschen, indem sie eine Vielzahl an Landschaftsfunktionen oder Ökosystemdienstleistungen bereitstellt.

Auch der derzeitige globale Klimawandel folgt der Logik des Systems „Mensch - räumliche Umwelt“. Der unbedachte, dauerhafte Ausstoß an klimawirksamen Treibhausgasen führt zu einer globalen Erwärmung, die in dieser Geschwindig- keit bisher unbekannt war (IPCC 2007). Der Mensch sieht sich konfrontiert mit den Folgen seines Handelns und versucht nun, um die Nachhaltigkeit des Gesamtsystems zu retten, das Klima durch weltweite Reduktion der Treibhausgasemissionen zu schützen. So bedeutsam derartige internationale Bemühungen zur Reduktion der Treibhausgasemissionen auch sind, darf nicht übersehen werden, dass das Klimasystem der Erde sehr träge ist und eine weitere Erwärmung mit sich bringen wird, auch dann, wenn es gelingen sollte, die Treibhausgasemissionen kurzfristig zu unterbinden. Vor diesem Hintergrund erscheint der gobale Klimawandel als unabwendbar. Fraglich ist vor allem, mit welchen konkreten Folgen in welchen Regionen zu rechnen ist. Die Beantwortung dieser Frage ist mit erheblichen Unsicherheiten verbunden, da sich einerseits die Intensität der zukünftigen Klimaänderungen aus den zukünftigen, derzeit noch unbekannten, Treibhausgasemissionen ergibt und andererseits davon abhängt, inwieweit die im globalen Maßstab indentifizierten Prozesse auf die regionale Ebene herunter-

Im Allgemeinen wird angenommen, dass die zukünftige weltweite Entwicklung der Treibhausgasemissionen von ökonomischen, demographischen und technologischen Faktoren abhängt, deren Entwicklung im Detail unbekannt ist. Um dennoch zukunftsbezogene Aussagen für Treibhausgasemissionen sowie die Folgen der Klimaerwärmung treffen gebrochen werden können. zu können, wurden vom Intergovernmental Panel on Climate Change (IPCC) sogenannte globale Emissionsszenarien definiert, die auf der Grundlage unterschiedlicher ökonomischer, demographischer und technologischer Entwicklungen ein breites Spektrum denkbarer zukünftiger Entwicklungen der genannten Faktoren wie auch der daraus resultierenden Treibhausgasemissionen aufzeigen und somit als Grundlage für regionale Klimamodelle herangezogen werden können (IPCC 2000). Unter anderem wurden folgende Szenarien definiert:

- A2: fortdauerndes Bevölkerungswachstum, heterogene ökonomische Entwicklung, kaum technologischer Wandel;

- A1B: mittleres Szenario, ökonomisches Wachstum, Bevölkerungswachstum bis 2050, ausgewogene Nutzung unterschiedlicher Energieformen;

I B1: konvergente globale Entwicklung, Bevölkerungswachstum bis 2050, technologischer Wandel mit hohem Anteil regenerativer Energienutzung. Auf dieser Grundlage wurden die Trends der Klimaerwärmung für die nächsten Dekaden bis zum Jahr 2100 berechnet, die zwar das Ausmaß der Klimaänderungen im globalen Maßstab deutlich machen, jedoch kaum räumlich differenzierte Aussagen für Europa zulassen (IPCC 2007).

\section{Der Klimawandel ist für die regionale Ebene relevant}

Aufgrund der geringen räumlichen Auflösung globaler Klimamodelle sind diese nicht unmittelbar für regionale Anpassungsstrategien verwendbar. Hierzu bedarf es spezifischer Regionalisierungsverfahren, die in Abhängigkeit von der Maßstabsebene sowie der räumlichen Lage und Ausdehnung des Betrachtungsgebietes jeweils wiederum mit Vor- und Nachteilen sowie mit Unsicherheiten behaftet sind.

Mithilfe statistischer und dynamischer Regionalisierungsverfahren kann von globalen Klimamodellen auf die regionale oder lokale Ebene geschlossen werden. 
Für die regionale Ebene eignet sich insbesondere das hydrostatische Modell REMO, mit dem eine räumliche Auflösung von zehn mal zehn Kilometern erreicht werden kann (Jacob/Podzun 1997). Die folgenden Analysen beruhen auf Berechnungen mit diesem Modell (Loibl et al. 2008). Eine Voraussetzung für die Auswahl von Klimaparametern auf der regionalen Ebene ist die Klärung der Frage, welche Klimafolgewirkungen vor dem Hintergrund planungsrechtlicher Kompetenzverteilungen auf dieser Ebene relevant sein können. Als relevant sind jene Wirkungen anzusehen, für die mit planerischen Instrumenten Maßnahmen der Anpassung formuliert und dargestellt oder festgesetzt werden können (Gruehn et al. 2008). Dabei handelt es sich einerseits um schleichende Veränderungen, andererseits um Extremereignisse:

I Zunehmender Verlust des Oberbodens durch Wassererosion,

- Steigende Gefährdung der Artenvielfalt,

I Zunehmende Schwankungen des Grundwasserspiegels,

- Einschränkung der als Brauchwasser nutzbaren Wasserressourcen,

I Häufigere Hitzeperioden oder Hitzewellen,
- Häufigere Starkregenereignisse und Sturzfluten,

- Veränderung von Frequenz und Stärke von Flusshochwässern,

】 Häufigere und höhere Sturmwasserstände,

- Steigende Gefahr von gravitativen Massenbewegungen,

- Steigende Waldbrandgefahr,

- Häufigere Beeinträchtigung und Zerstörung der Infrastruktur.

Die vorstehenden potenziellen Wirkungen werden im Wesentlichen durch die Änderungen folgender Klimaparameter ausgelöst:

- Anzahl der Frosttage,

- Anzahl der Hitzetage,

- Anzahl der Tage mit Starkniederschlägen,

- Sommerniederschlag,

- Winterniederschlag,

- Jahresmitteltemperatur.

\section{Folgen des Klimawandels in deutschen Regionen}

Wie oben aufgezeigt, können mehrere Klimaelemente Auslöser für Klimafolgewirkungen auf der regionalen Ebene sein. Aus Berechnugnen des REMO-Modells wird deutlich, dass es sich keines-

Abbildung 1: Klimawandelregionstypen für den Zeitraum 2071-2100 in Abhängigkeit von unterschiedlichen Emissionsszenarien

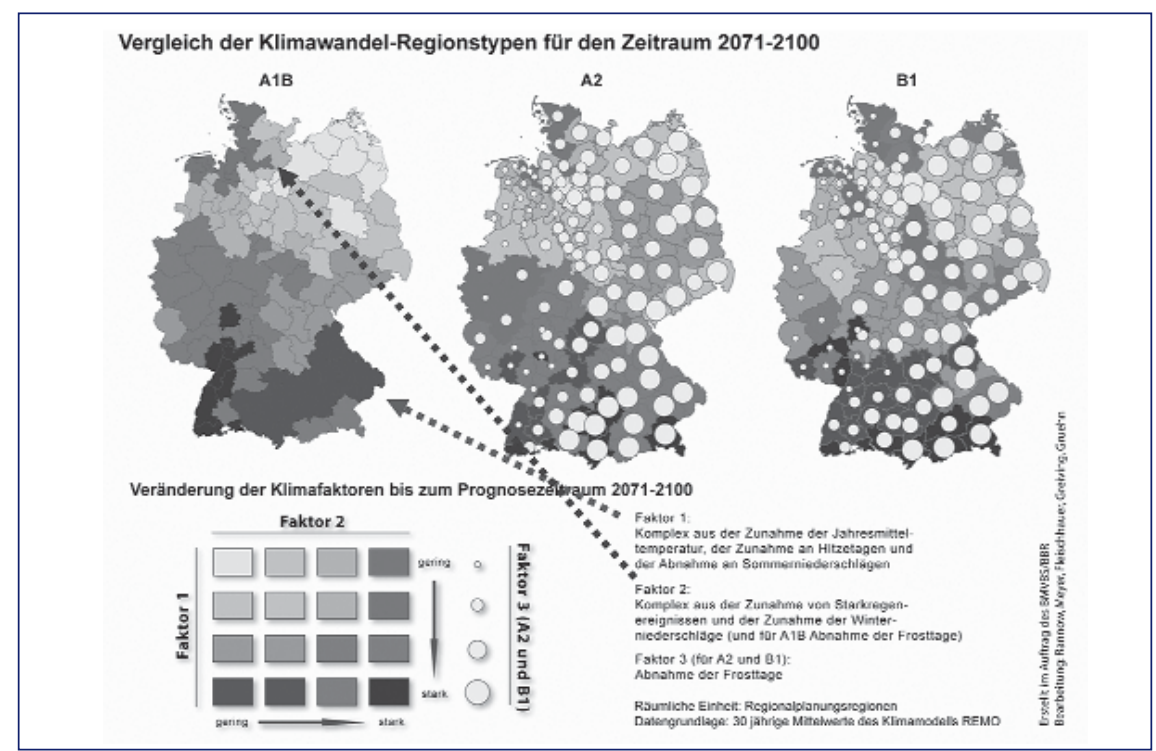

Quelle: Gruehn et al. 2008

wegs um eine deutschlandweit einheitliche Erwärmung handelt, sondern dass regionalspezifische Veränderungen zu verzeichnen sind. Je nach dem zugrunde gelegten Emissionsszenario, herrschen andere Verteilungsmuster der Klimaänderungen vor. Das Gleiche gilt für andere Klimaparameter. Auf der Grundlage der hier betrachteten drei Emissionsszenarien, drei Prognosezeiträume sowie sechs ausgewählten Klimaparameter ergeben sich 54 spezifische räumliche Verteilungsmuster (Gruehn et al. 2008). Um die Komplexität dieser Zusammenhänge unter anderem für Entscheidungsträger besser verständlich $\mathrm{zu}$ machen, wurde mithilfe einer Hauptkomponentenanalyse die Komplexität reduziert. Dabei werden die sechs Klimaeingangsparameter je nach Szenario zu zwei (Szenario A1B) beziehungsweise drei Faktoren (Szenario A2, B1) zusammengefasst, auf deren Grundlage so genannte Klimawandelregionstypen dargestellt werden können. In Abbildung 1 ist dies für den Zeitraum 2071-2100 dargestellt.

Auf der Basis von Abbildung 1 kann verglichen werden, wie sich die unterschiedlichen Szenarienannahmen auf bestimmte Regionen auswirken. Zudem wird gezeigt, welche durch die Faktoren repräsentierten Klimaparameter sich in welchen Regionen wie stark auswirken. Schließlich können kumulative Effekte sichtbar gemacht werden, wenn beispielsweise in Szenario A1B für das Oberrheintal Veränderungen beider Klimafaktoren indiziert werden, während an der Nordseeküste vor allem Faktor 2 und in großen Teilen Süddeutschlands Faktor 1 bedeutsam ist. Insgesamt zeigt es sich, dass mit einer Veränderung der Klimafaktoren vor allem im Westen und Süden Deutschlands zu rechnen ist.

Die potenzielle Betroffenheit einer Region ist jedoch nicht nur von der Änderung der Klimaparameter aufgrund des Klimawandels abhängig, sondern auch von der Anfälligkeit des Raumes gegenüber der Änderung spezifischer Klimaparameter. Beispielsweise ist die Anfälligkeit von Regionen mit sehr erodiblen Böden gegenüber einer Zunahme an Starkregenereignissen höher als in Regio- 
nen, die kaum erodible Böden aufweisen. Für die spezifischen raumordnungsrelevanten Wirkfolgen des Klimawandels im Sinne der oben skizzierten schleichenden Veränderungen und Extremereignisse können unter Berücksichtigung der beiden oben genannten Eingangsparameter die Betroffenheiten des Raumes bestimmt werden (Gruehn et al. 2008). Werden die zunächst singulären Betroffenheiten räumlich überlagert, ergeben sich kumulative Effekte, die insgesamt eine Ungleichverteilung der räumlichen Betroffenheit gegenüber dem Klimawandel indizieren.

\section{Klimaanpassungsstrategien auf regionaler Ebene sind notwendig}

Wie die vorstehenden Ausführungen zeigen, weisen die zu erwartenden Klimaänderungen, die Anfälligkeit des Raumes sowie die Betroffenheit des Raumes gegenüber spezifischen Klimaänderungen eine hohe raum-zeitliche Variabilität auf. Dies spricht in hohem Maße dafür, auf der regionalen Ebene in Kooperation mit den jeweiligen Akteuren, regionsspezifische Anpassungsstrategien zu entwickeln. Auch aus diesem Grunde hat das BBSR die Durchführung zahlreicher Modellprojekte initiiert, die sich auf der Grundlage der hier dargestellten Ergebnisse mit der Erarbeitung regionaler Klimaschutz- und -anpassungsstrategien befassen (Baumüller et al. 2010). Gleichwohl stellt sich die Frage, ob, bezogen auf die oben angesprochenen Funktionen der Landschaft, auch allgemeine Prinzipien der Klimaanpassung formuliert werden können.

Geht man von den in Abbildung 1 dargestellten Änderungen der Klimafaktoren beziehungsweise Kimaparameter aus, zeigen sich trotz hoher räumlicher Variabilität der einzelnen Parameter grundsätzliche Trends der Klimaentwicklung. Zwar sind einzelne Faktoren in spezifischen Regionen unterschiedlich ausgeprägt. Die grundsätzliche Richtung der Änderung bleibt jedoch gleich: Es geht in jedem Fall um eine Zunahme von Jahresmitteltemperatur, Hitzetagen, Starkrege- nereignissen und Winterniederschlägen und eine Abnahme von Sommerniederschlägen und Frosttagen. Lediglich der Grad der Ausprägung der Parameter in den unterschiedlichen Regionen variiert.

Um den mit dem Klimawandel verbundenen Wirkungen zu begegnen oder sie zu vermindern, sind spezifische Flächentypen und Funktionsräume denkbar. Diese Flächentypen reichen von bioklimatisch wirksamen Kühlflächen bis hin zu spezifischen Habitaten. Sie alle haben eines gemeinsam: Sie stellen sehr effektive Puffer- beziehungsweise Speicherflächen im Naturhaushalt dar und minimieren die mit dem Klimawandel verbundenen Risiken. Klassischerweise handelt es sich bei den genannten Flächentypen um Kategorien, die bereits seit Jahrzehnten im Rahmen der Landschaftsplanung als schutzwürdige Bereiche vorgeschlagen und dargestellt werden. Wie wissenschaftliche Erfolgskontrollen belegen, werden die Darstellungen der Landschaftsplanung im Rahmen gesamtplanerischer Abwägungen oft nur teilweise berücksichtigt (Gruehn 1998). Dies begünstigt folglich die Umwidmung von Flächen, die aufgrund ihrer beträchtlichen Speicheroder Pufferwirkung eine hohe Bedeutung für die Anpassung an den Klimawandel haben in Kategorien mit minderer Bedeutung. Als Beispiel können unter anderem die Verkleinerung von Retentionsräumen durch bauliche Entwicklung im Außenbereich oder die städtebauliche Verdichtung im Innenbereich zu Lasten bioklimatisch bedeutsamer Kühlflächen genannt werden. Wie das Bundesamt für Naturschutz gezeigt hat, sind große Teile der Flusslandschaften Deutschlands in den vergangenen Jahrzehnten durch zum Teil extremen Verlust von Überschwemmungsflächen gekenzeichnet (BfN 2009).

Ein weiteres Problem der aktuellen Raumentwicklung ist, dass die Neuanweisung von Baugebieten teilweise noch immer in Bereiche gelenkt wird, die sich durch eine hohe biotische Ertragsfunktion auszeichnen. Mit dem Verlust dieser Standorte wird insgesamt zu einer Beschleunigung des Abflusses beigetragen, da die wasserspeichernde Wirkung des
Bodens nicht mehr zur Abflussverzögerung beitragen kann.

Die aufgezeigten Trends der Raumentwicklung scheinen einer erfolgreichen Implementation bisheriger Ansätze von Klimaanpassungsstrategien tendenziell entgegenzuwirken. Ein Festhalten am gesamtplanerischen Abwägungsgebot, das ökologisch bedeutsame Flächen beliebig verhandelbar macht, sollte daher in Frage gestellt werden.

\section{Literatur}

Baumüller, J. / Baumüller, N.: „Städte im Klimawandel“. In: Planerln 2/2010. S. 17-20.

BfN - Bundesamt für Naturschutz: Verlust von Überschwemmungsflächen. Bonn 2009.

Gruehn, D.: Zur Berücksichtigung der Belange von Naturschutz und Landschaftspflege in der vorbereitenden Bauleitplanung. Europäische Hochschulschriften 42 (22). 1998.

Gruehn, D. et al.: Raumentwicklungsstrategien zum Klimawandel - Vorstudie für Modellvorhaben. BBR-Online-Publikation 19/2008. Bonn.

IPCC - Intergovernmental Panel on Climate Change: Emissions Scenarios. Cambridge University Press. 2000.

IPCC- Intergovernmental Panel on Climate Change: Working Group II Report „Impacts, Adaptation and Vulnerability“. Cambridge University Press. 2007.

Jacob, D. / Podzun, R.: Sensitivity studies with the regional climate model REMO. In: Meteor. Atmos. Phys. 63 (1-2/1997). S. 119-129.

Langer, H.: Mensch und Umwelt als ökologisches System. In: Buchwald, K. / Engelhardt, W. (Hrsg.): Handbuch für Planung, Gestaltung und Schutz der Umwelt (1). 1978. S. 134-146.

Loibl, W. et al.: Modellvorhaben - Raumentwicklungsstrategien zum Klimawandel - Räumliche Analyse der Signale des Klimawandels für die Planungsregionen Deutschlands Vergleich ausgewählter Modellergebnisse, Extraktion wesentlicher Indikatoren. ARC-sys-Berichte 0167/2008. Wien.

I AUTOR + KONTAKT

Dr. Dietwald Gruehn ist Professor für Landschaftsökologie und Landschaftsplanung sowie geschäftsführender Leiter des Instituts für Raumplanung an der Technischen Universität Dortmund.

TU Dortmund, 44221 Dortmund. Tel.: ++49 231 755-2285,

E-Mail: dietwald.gruehn@tu-dortmund.de 
(c) 20I0 Authors; licensee IÖW and oekom verlag. This is an article distributed under the terms of the Creative Commons Attribution Non-Commercial No Derivates License (http://creativecommons.org/licenses/by-nc-nd/3.o/), which permits unrestricted use, distribution, and reproduction in any medium, provided the original work is properly cited. 